\title{
INVERSIÓN PRIVADA E IMPUESTOS CORPORATIVOS: EVIDENCIA PARA ChILE*
}

\author{
RODRIGO CERDA* \\ FELIPE LARRAÍN \\ Pontificia Universidad Católica de Chile
}

Using annual data from Chile since the beginning of the eighties, we show that an increase in the corporate tax reduces firm's investment. However the impact differs across firm size. In small and medium sized firms, investment as a fraction of the capital stock declines between $0.5 \%$ and $1.6 \%$ while on large corporations the impact is much less significant.

\section{$J E L: \mathrm{H} 25, \mathrm{H} 32$}

Keywords: Investment, Small Size Firms, Corporate Taxation

\section{INTRODUCCIÓN}

El impacto de la tributación sobre el comportamiento de una empresa ha sido el foco de muchas investigaciones en economía. En particular, el efecto de la política tributaria a nivel corporativo sobre el nivel de equilibrio del stock de capital y el momento óptimo de inversión es un tema de mucho interés para los economistas. Desde las tempranas contribuciones de Jorgenson (1963), Hall y Jorgenson (1967) y Tobin (1969) existe un consenso relativamente amplio entre los economistas en el hecho de que las tasas de impuesto corporativo más altas tienden a reducir la inversión y el stock de capital de largo plazo. La teoría ha enfatizado la importancia de la tributación sobre la inversión corporativa. La teoría $q$ de Tobin (1969) divide los determinantes de las decisiones de inversión en tres

\footnotetext{
*Agradecemos la valiosa colaboración en la investigación de Francisco Parro y Se Kyu Choi. Recibimos útiles comentarios de Juan Eduardo Coeymans, de un árbitro anónimo y de los participantes en el seminario interno del Departamento de Economía de la PUC realizado en enero de 2005. Cualquier error restante es de nuestra responsabilidad

**E-mails: rcerda@faceapuc.cl, flarrain@faceapuc
} 
componentes: el mercado accionario, la política de impuestos a la inversión y los costos de ajustes. La teoría del costo de uso del capital (user-cost theory) desarrollada por Jorgenson (1963) y extendida por Hall y Jorgenson (1967) indica a la tributación corporativa como determinante central del costo del capital.

Este consenso que nace de la teoría económica no es ampliamente compartido a nivel empírico, especialmente en lo que concierne a la magnitud del impacto de los impuestos corporativos sobre la inversión. Además, los estudios empíricos han evolucionado desde el uso de datos agregados a la utilización de datos microeconómicos. Aunque estos últimos son de mayor riqueza, con lo cual potencialmente mejoran el análisis empírico, generalmente no están disponibles en el caso de los países en desarrollo. Sin embargo, la evidencia respecto de los países en desarrollo es un tópico muy interesante dadas las grandes restricciones para endeudarse que usualmente enfrentan las empresas en dichos países.

Este trabajo intenta llenar parte de este vacío presentando evidencia acerca del impacto de la tributación corporativa sobre la tasa de inversión con datos para Chile desde 1981 a 1996. Nos focalizamos en Chile debido a las interesantes características de los datos y también del periodo analizado. En dicho periodo, la tasa de impuesto corporativo varió ampliamente, desde casi 50\% al inicio a $0 \%$ en 1990 (las utilidades repartidas pagaban una tasa de impuesto de $10 \%$, mientras que las utilidades retenidas pagaban una tasa de $0 \%$ ), estabilizándose en $15 \%$ al final de nuestra muestra.

Usamos datos microeconómicos de una serie de encuestas chilenas sobre empresas manufactureras desde 1981 a 1996. Nuestra muestra contiene compañías de diverso tamaño, lo que nos permite estudiar si el impacto de los impuestos corporativos difiere dependiendo del tamaño de la empresa. La muestra incluye pequeñas y medianas empresas, conocidas como PYMEs (aquellas con ventas anuales entre US\$72,500 y US\$3.0MM, aproximadamente) y también compañías grandes (ventas sobre US\$3.0MM). Las PYMEs usualmente enfrentan restricciones para endeudarse, lo cual constituye una importante motivación para analizarlas en detalle. Bustos, Engel y Galetovic (2004) -BEG- también estudiaron este tema analizando datos microeconómicos para Chile. Nuestro estudio difiere del de Bustos et al. en que ellos incluyen en su base de datos sólo sociedades anónimas abiertas, cuyo tamaño es considerablemente mayor al de una firma promedio de la economía; estas grandes compañías generalmente no enfrentan restricciones crediticias. Tal como señalamos más adelante, esta característica de nuestra base de datos puede explicar resultados muy diferentes a los obtenidos por ellos.

Explotaremos la riqueza de nuestros datos en diversas formas. Por una parte, la gran variación en la tasa de impuesto corporativo nos permitirá averiguar el impacto de la tributación corporativa sobre las decisiones de inversión de las firmas. Presentamos resultados derivados de estimaciones con efectos fijos y, luego, estimaciones usando paneles dinámicos de acuerdo a la metodología de Arellano-Bond.

Este trabajo se organiza como sigue. La sección 2 revisa la literatura empírica. Luego, la sección 3 describe los datos y discute la metodología, mientras que la sección 4 analiza los resultados. Finalmente, la sección 5 concluye. 


\section{REVISIÓN DE LA LiTERATURA}

A nivel teórico, la mayoría de los modelos indica que tasas de impuesto más altas reducen el stock deseado de capital. En el modelo neoclásico (Jorgenson, 1963) la inversión se basa en un comportamiento optimizador de las firmas, sujeto a la trayectoria de las tasas de interés, los precios del capital y las políticas tributarias. En la solución de este problema, la inversión responde negativamente a la tasa real de costo del capital, la cual es una función creciente de la tasa de interés, la tasa de impuesto corporativo y la tasa de depreciación, y positivamente a las distintas modalidades de depreciación y a los subsidios a la inversión, entre otras variables. Intuitivamente, el impacto negativo que produce un incremento en la tasa de impuesto corporativo sobre la inversión se debe a la disminución del producto marginal futuro después de impuesto de la inversión actual.

De acuerdo a Tobin (1969), la decisión de cuánto invertir se basa en la rentabilidad esperada; por tanto, la tasa de inversión depende del cuociente entre el valor de mercado de los activos de una empresa y su valor de reposición. Este cuociente se conoce usualmente como $q$. En este caso, una tasa de impuesto corporativo más alta reduce el valor de mercado después de impuestos de los activos de la empresa y, por consiguiente, disminuye el cuociente $q$ y la tasa de inversión.

A pesar de la fuerte evidencia a nivel teórico favoreciendo una relación negativa entre la tasa de impuesto corporativo y la inversión, existen casos en que es posible que el stock de capital deseado aumente con una mayor tasa de impuesto. ${ }^{1}$ Este hecho explica por qué la literatura reciente se ha focalizado en las estimaciones empíricas.

A finales de los 60s, las estimaciones empíricas que emergieron del modelo neoclásico - usando datos agregados- no fueron concluyentes. El uso de este tipo de datos fue una clara limitación al momento de distinguir entre movimientos de la inversión producto del ciclo económico o de cambios en la política tributaria, entre otras variables, ya que un número de variables agregadas tienden a moverse juntas durante el ciclo económico, haciendo difícil aislar los efectos de variables fundamentales individuales sobre la inversión.

La teoría $q$ de Tobin ha sido estimada usando mínimos cuadrados en base a datos agregados ( Summers, 1981) o un panel de empresas (Fazzari, Hubbard y Peterson, 1988). Típicamente, las estimaciones asumen la existencia de costos de ajuste (generalmente cuadráticos) en la función de inversión. Los resultados empíricos son raramente consistentes con los supuestos del modelo teórico. Tales resultados pueden indicar tanto un rechazo de la teoría $q$ como la existencia de errores de medición en el cuociente $q$, evidencia de una correlación negativa entre los costos de ajustes y el cuociente $q$ ajustado por impuestos o, simplemente, el rechazo al supuesto de la forma funcional cuadrática. Adicionalmente, se debe señalar que el cuociente $q$ ajustado por impuestos explica la inversión algo mejor

\footnotetext{
${ }^{1}$ Ver Bustos et al. (2004).
} 
que el cuociente $q$ sin ajustar, especialmente para los fuertes ajustes impositivos asociados a los cambios en las leyes sobre impuestos al ingreso corporativo (Cummins, Hassett y Hubbard, 1994). Esto nos indicaría que el error de medición de la variable $q$ es serio y que se puede detectar más fácilmente el impacto de $q$ sobre la inversión cuando el impacto de la tasa de impuestos sobre $q$ es más importante.

Desde los 60 hasta mediados de los 80 , siguiendo la contribución seminal de Modigliani y Miller (1958) -la irrelevancia de la estructura financiera para las decisiones de inversión real- la literatura no ha distinguido la decisión de inversión entre firmas restringidas financieramente y no restringidas. Sin embargo, después del estudio de Fazzari, Hubbard y Petersen (1988), las investigaciones empíricas han enfatizado la importancia de las restricciones financieras sobre las decisiones de inversión moviéndose, por consiguiente, más allá del supuesto de una firma representativa, y clasificando a las firmas en la categoría de "alto valor" y "bajo valor". Estudios empíricos han mostrado que la disponibilidad de fondos internos afecta la inversión (Hubbard et al., 1995). Por lo tanto, es probable que el impacto de los impuestos corporativos sobre la inversión de las firmas que enfrentan restricciones crediticias sea mayor al predicho por el enfoque neoclásico. Este escenario es particularmente relevante para los países en desarrollo, cuyos mercados financieros están menos desarrollados y, por consiguiente, es probable que presenten restricciones crediticias.

Los estudios de inversión en Chile son recientes. Medina y Valdés (1998) usan datos microeconómicos para Chile de firmas transadas en la Bolsa para analizar la importancia de los flujos de caja sobre las decisiones de inversión de una firma. Sus resultados están en línea con la idea de que la disponibilidad de fondos internos importa para las decisiones de inversión más allá de la rentabilidad del proyecto. Aunque ellos no verifican directamente la relación entre impuestos corporativos e inversión, sus resultados sugieren que los impuestos sobre las utilidades retenidas reducen la inversión.

Hsieh y Parker (2002) presentan evidencia que señala a la reforma tributaria a nivel corporativo como una causa directa del boom de inversión en Chile. Específicamente, ellos usan datos agregados, a nivel de industrias y plantas, para mostrar que la reducción de los impuestos sobre las utilidades retenidas en 19841986 aumentaron la disponibilidad de fondos internos para empresas restringidas financieramente, incrementando, por consiguiente, la inversión en dichas compañías. La intuición de estos resultados es que en países con mercados financieros pobremente desarrollados, tal como era el caso de Chile en 1984, los impuestos sobre las utilidades retenidas reducen fondos internos en las firmas con restricciones financieras y, por consiguiente, disminuyen la inversión.

Bustos et al., (2004) estudian los procesos de inversión usando un panel con datos anuales de 83 empresas para el periodo 1985-1995, mostrando que el impacto de la tasa de impuestos corporativos sobre el stock de capital de largo plazo no es significativo. Sin embargo, la base de datos que ellos utilizan incluye sólo sociedades anónimas abiertas, cuyo tamaño es considerablemente mayor al 
de una empresa promedio en Chile y, por consiguiente, éstas no presentarían restricciones financieras.

Vergara (2004) investiga empíricamente la relación entre la reforma tributaria de los 80 y el desempeño de la inversión en Chile desde dicha reforma. Usando datos agregados para el periodo 1975-2003, sus resultados indican que la reforma tributaria explica un incremento de la inversión privada de $3 \%$ del PIB. Por otra parte, a base de datos microeconómicos de 87 compañías transadas en Bolsa para el periodo 1980-2002 se confirma la evidencia de que la inversión fue positivamente afectada por la reforma tributaria.

En resumen, existen algunos estudios que analizan el impacto de los impuestos corporativos sobre la inversión para el caso de Chile. La contribución de nuestro estudio radica en el análisis de una muestra extensa que incluye pequeñas, medianas y grandes empresas. Nuestro estudio también utiliza diferentes metodologías econométricas: presentamos estimaciones con efectos fijos y paneles dinámicos usando la metodología de Arellano y Bond (1991). El siguiente cuadro resume los principales estudios de inversión en Chile, incluyendo el nuestro $^{2}$, y sus respectivas diferencias metodológicas:

CUADRO 1

PRINCIPALES ESTUDIOS DE INVERSION EN CHILE

\begin{tabular}{|c|c|c|c|c|}
\hline & Hsieh y Parker & $\begin{array}{l}\text { Bustos, Engel y } \\
\text { Galetovic }\end{array}$ & Vergara & Cerda y Larraín \\
\hline $\begin{array}{l}\text { Fuente de la } \\
\text { muestra }\end{array}$ & ENIA & FECUS & FECUS & ENIA \\
\hline Sectores & Manufacturas & Todos & Todos & Manufacturas \\
\hline Tamaño & Todas & Grandes & Grandes & Todas \\
\hline Período & $1982-1990$ & 1985-1995 & $1980-2002$ & 1981-1996 \\
\hline $\begin{array}{l}\text { Método de } \\
\text { estimación }\end{array}$ & Panel & $\begin{array}{c}\text { Mínimos Cuadrados } \\
\text { Ponderados }\end{array}$ & $\begin{array}{l}\text { Panel con } \\
\text { Efectos Fijos }\end{array}$ & $\begin{array}{l}\text { Panel con Efectos } \\
\text { Fijos y Paneles } \\
\text { Dinámicos } \\
\text { Arellano-Bond }\end{array}$ \\
\hline $\begin{array}{l}\text { Estrategia de } \\
\text { modelamiento }\end{array}$ & $\begin{array}{l}\text { Modelo de forma } \\
\text { reducida }\end{array}$ & $\begin{array}{l}\text { Modelo estructural, } \\
\text { optimización } \\
\text { intertemporal }\end{array}$ & $\begin{array}{l}\text { Modelo de forma } \\
\text { reducida }\end{array}$ & $\begin{array}{l}\text { Modelo de forma } \\
\text { reducida }\end{array}$ \\
\hline $\begin{array}{l}\text { Principales } \\
\text { resultados }\end{array}$ & $\begin{array}{c}\text { Efecto significativo } \\
\text { en firmas } \\
\text { restringidas } \\
\text { financieramente }\end{array}$ & $\begin{array}{l}\text { Efecto pequeño y } \\
\text { positivo en algunos } \\
\text { años }\end{array}$ & $\begin{array}{c}\text { Efecto } \\
\text { significativo }\end{array}$ & $\begin{array}{l}\text { Efecto significativo } \\
\text { en pequeñas y } \\
\text { medianas empresas }\end{array}$ \\
\hline
\end{tabular}

Nota: ENIA es una encuesta chilena de la industria manufacturera, mientras que FECUS es una base de datos que incluye empresas transadas en Bolsa.

\footnotetext{
${ }^{2}$ Agradecemos al árbitro anónimo por sugerirnos la inclusión de este cuadro.
} 


\section{Datos y Metodología}

En esta sección discutimos las propiedades de nuestra base de datos y las posibilidades de estimación. Para estudiar los efectos de los impuestos sobre las decisiones de inversión de las empresas chilenas, analizamos datos desagregados de la ENIA, encuesta chilena de la industria manufacturera, para el periodo 1981-1996. La riqueza de los datos nos permite estimar la dinámica de la inversión dentro de cada firma por un periodo considerable de tiempo. Considerando la dispersión de las tasas de impuesto a través de los años que abarcan nuestro análisis, podemos extrapolar el efecto de diferentes tasas de impuestos controlando por todas las características de la economía chilena.

CUADRO 2

PYMES Y CORPORACIONES

\begin{tabular}{lcccccc}
\hline & $\begin{array}{c}(1) \\
\text { Micro } \\
\text { empresas }\end{array}$ & $\begin{array}{c}(2) \\
\text { Empresas } \\
\text { pequeñas }\end{array}$ & $\begin{array}{c}(3) \\
\text { Empresas } \\
\text { medianas }\end{array}$ & $\begin{array}{c}(4) \\
\text { PYMEs }\end{array}$ & $\begin{array}{c}(5) \\
\text { Empresas } \\
\text { grandes }\end{array}$ & $\begin{array}{c}(6) \\
\text { Total }\end{array}$ \\
\hline Número de empresas & 535.338 & 93.923 & 13.164 & 107.087 & 6.066 & 648.491 \\
\% del total & 82.5 & 14.5 & 2.0 & 16.5 & 1.0 & 100 \\
\% de las ventas & 3.7 & 10.1 & 10.1 & 20.2 & 76.1 & 100 \\
Ventas promedio en UFs & 456 & 7.161 & 51.096 & 12.562 & 836.395 & 10.274 \\
Empleo (\%) Corfo & 38.7 & 35.2 & 12.5 & 47.7 & 9.7 & 100 \\
Empleo (\%) CASEN & 40.4 & 36.6 & 13.0 & 49.6 & 10.1 & 100 \\
\hline
\end{tabular}

Fuente: Cabrera, de la Cuadra, Galetovic y Sanhueza (2002). En el cuadro, UF denota Unidades de Fomento, una unidad monetaria chilena indexada a la inflación. Los datos de ventas y número de firmas provienen del SII. Microempresas son aquellas con ventas anuales hasta UF 2.400 (US\$ 72.500) por año, mientras que las pequeñas y medianas empresas son aquellas con ventas anuales entre UF 2.400-25.000 (US\$ 72.500-754.000) y UF 25.000-100.000 (US\$ 754.000-3.000.000). Las empresas grandes son aquellas con ventas anuales mayores a UF 100.000. PYME denota pequeña y mediana empresa. Los datos de empleo se obtuvieron de CORFO y de la encuesta CASEN de octubre de 2000.

Nuestros datos a nivel microeconómico nos permiten obtener resultados para firmas de diferente tamaño. Tal como señalamos anteriormente, esto constituye un factor relevante en la literatura, dada la existencia de restricciones financieras. Estamos interesados en explorar esta distinción debido a la gran heterogeneidad presente entre compañías en la economía chilena. El Cuadro 2 presenta la descripción de empresas chilenas según tamaño -de acuerdo a la clasificación del Servicio de Impuestos Internos, SII-. El cuadro muestra que mientras las empresas grandes representan un gran porcentaje de las ventas totales, emplean sólo al 10\% del trabajo total y significan sólo un $1 \%$ del número total de firmas. Las pequeñas y medianas empresas (PYMEs) representan un $20 \%$ de las ventas totales y un $50 \%$ del empleo total. 
La fuente de nuestros datos microeconómicos es la Encuesta Nacional Industrial Anual (ENIA), compilada sobre firmas manufactureras por el Instituto Nacional de Estadísticas (INE) a finales de cada año. Nuestra base de datos contiene información sobre ventas, inversión, valor agregado, ubicación y clasificación en el sector de manufacturas (a 4 dígitos de acuerdo a la clasificación ISIC), entre otras variables, para un número aproximado de 970 compañías. Las empresas incluidas en nuestra base de datos son muy heterogéneas, tal como se puede apreciar en los cuadros 3 y 4 . El Cuadro 3 describe las compañías según tamaño en 1996; tal como se ve en el cuadro, nuestros datos se concentran en PYMEs y grandes compañías. De hecho, la ENIA incluye sólo empresas que emplean al menos 10 trabajadores en el año de la encuesta; esto excluye microempresas, las que representan cerca del 3,7\% de las ventas totales y un $40 \%$ del empleo total de Chile. Adicionalmente, la ENIA incluye sólo información sobre empresas manufactureras, sector que representa alrededor del 16\% del empleo total de la economía chilena. Sin embargo, el sector manufacturero es de especial interés dada la heterogeneidad en cuanto a tamaño de las compañías y subsectores en la muestra. El Cuadro 4 describe las firmas de acuerdo al número de trabajadores. Como puede verse, sobre un $95 \%$ de las firmas contrata menos de 500 trabajadores. Nuestra base de datos también considera, sin embargo, 25 empresas por año que contratan más de 500 trabajadores y un promedio de sólo 6 a 7 empresas por año con mil o más trabajadores.

CUADRO 3 EMPRESAS SEGUN TAMAÑO, 1996

\begin{tabular}{lcccccc}
\hline & Micro & Pequeñas & Medianas & PYME & Grandes & Total \\
\hline $\begin{array}{l}\text { Ventas anuales } \\
\text { (en miles de UF) }\end{array}$ & $($ menos de 2.4) & $\begin{array}{c}\text { (entre 2.4 } \\
\text { y 25 UF) }\end{array}$ & $\begin{array}{c}\text { (entre 25 } \\
\text { y 100) }\end{array}$ & & (sobre 100) & \\
Número de empresas & 7 & 332 & 275 & 607 & 366 & 973 \\
$(\%$ del total) & $0.7 \%$ & $34.1 \%$ & $28.3 \%$ & $62.4 \%$ & $37.6 \%$ & $100 \%$ \\
\hline
\end{tabular}

Fuente: Cálculos propios, usando información de la encuesta ENIA 1996.

CUADRO 4

DESCRIPCION DE EMPRESAS SEGUN NUMERO DE TRABAJADORES, ENIA, VARIOS AÑOS

\begin{tabular}{|c|c|c|c|c|c|c|c|c|c|}
\hline \multicolumn{10}{|c|}{ Tamaño de las empresas según número de trabajadores } \\
\hline \multirow[b]{2}{*}{$\begin{array}{c}\text { Tamaño } \\
\text { (número de trabajadores) }\end{array}$} & \multicolumn{3}{|c|}{ Todos los años } & \multicolumn{3}{|c|}{1985} & \multicolumn{3}{|c|}{1996} \\
\hline & Frec. & $\%$ & $\begin{array}{l}\text { Frec. } \\
\text { acum. }\end{array}$ & Frec.. & $\%$ & $\begin{array}{l}\text { Frec. } \\
\text { acum }\end{array}$ & Frec. & $\%$ & $\begin{array}{l}\text { Frec. } \\
\text { acum }\end{array}$ \\
\hline 10 a 19 & 3.768 & 22.78 & 22.78 & 255 & 26.21 & 26.21 & 214 & 21.99 & 21.99 \\
\hline 20 a 49 & 6.420 & 38.81 & 61.59 & 399 & 41.01 & 67.21 & 325 & 33.40 & 55.4 \\
\hline 50 a 99 & 2.655 & 16.05 & 77.64 & 142 & 14.59 & 81.81 & 166 & 17.06 & 72.46 \\
\hline 100 a 199 & 1.716 & 10.37 & 88.02 & 88 & 9.04 & 90.85 & 124 & 12.74 & 85.2 \\
\hline 200 a 499 & 1.429 & 8.64 & 96.66 & 65 & 6.68 & 97.53 & 101 & 10.38 & 95.58 \\
\hline 500 a 999 & 405 & 2.45 & 99.11 & 18 & 1.85 & 99.38 & 35 & 3.60 & 99.18 \\
\hline$>1000$ & 1.48 & 0.89 & 100 & 6 & 0.62 & 100 & 8 & 0.82 & 100 \\
\hline
\end{tabular}

Fuente: Cálculos propios usando información de la encuesta. 
Para construir las variables de interés, seguimos la teoría neoclásica de inversión asumiendo que la inversión deseada queda determinada al igualar el retorno marginal de la inversión a su costo financiero, $r_{t}$ :

$$
\operatorname{Prof}_{t}=V M P K_{t}-\delta \leq r_{t}
$$

donde $V M P K_{t}$ es el valor del producto marginal del capital y $\delta$ es su tasa de depreciación. La condición (1) se mantiene con igualdad si la inversión deseada es positiva. Si la función de producción de las firmas (o de la economía) es del tipo Cobb-Douglas, podemos escribir (1) como sigue: ${ }^{3}$

$$
\frac{P_{t}^{y}}{P_{t}^{k}} \frac{\alpha\left(1-\tau_{1}\right) Y_{t}}{K_{t}}-\delta \leq r_{t}
$$

Donde $\alpha Y_{t} / K_{t}$ es el producto marginal del capital ( $\alpha$ es la participación del capital en la producción) mientras que $P_{t}^{Y} / P_{t}^{K}$ mide el valor del producto marginal del capital en unidades de stock de capital, y $\tau_{t}$ denota la tasa de impuesto corporativo. De (2) podemos definir la siguiente función implícita de los determinantes de la inversión deseada de una firma:

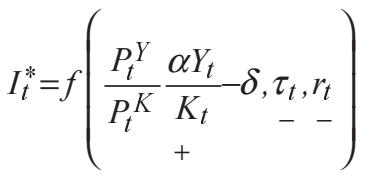

Nuestras estimaciones se basarán en la ecuación (3). Sin embargo, debemos hacer algunas aclaraciones. Primero, tal como se puede apreciar en (3), separamos el efecto del valor del producto marginal del impacto de la tasa de impuesto. Hacemos esto, pues pudiesen haber casos de restricciones crediticias en los cuales las empresas no pueden obtener los fondos necesarios para financiar la inversión deseada a la tasa $\tau_{t}$. Por consiguiente, para un valor dado del producto marginal del capital, el impacto de cambios de la tasa de impuestos puede ser muy diferente si consideramos firmas que deben financiar la inversión usando fondos internos. Segundo, la condición presentada anteriormente se refiere a la inversión deseada. Como es usual en la literatura, debemos considerar la existencia de costos de ajuste. Por lo tanto, estimaremos modelos dinámicos que consideran rezagos en el impacto de nuestras variables exógenas sobre la inversión.

En nuestras estimaciones, la variable dependiente es la inversión como fracción del stock de capital para cada empresa en cada año. Usamos como variable explicativa específica empresa/año, la rentabilidad del capital $\left(\operatorname{Prof}_{i t}\right)$, la cual definimos como $\operatorname{Prof}_{i t}=P_{t}^{Y} / P_{t}^{K} \alpha Y_{i t} / K_{i t}-\delta_{i t}$, donde el subíndice $i$ indica empresa y $t$ indica año, la variable $Y_{i t}$ es el valor agregado adicionado por cada empresa, $K_{i t}$ es el stock de capital por empresa, mientras que $P_{t}^{Y} / P_{t}^{K}$, el precio del producto relativo al precio del capital, es medido por los deflactores obtenidos de

${ }^{3}$ Un enfoque similar fue usado por Coeymans (2001), Du Toit y Moolman (2004) y Phelps (1962), entre otros 
las cuentas nacionales. Para construir esta variable, usamos una participación del capital $\alpha=0.4$. Para construir la tasa de depreciación, la cual también es variante entre firmas y en el tiempo, seguimos el enfoque de Bustos et al. (2004) asumiendo que la tasa de depreciación varía entre los distintos tipos de capital. Específicamente, asumimos que las obras de construcción se deprecian a una tasa de $2 \%$ por año, mientras que la maquinaria y vehículos se deprecian a una tasa de $8 \%$ y $15 \%$ por año, respectivamente. Dado que en nuestros datos conocemos cada tipo de capital por firma, computamos la depreciación promedio año por año ponderando dichas tasas de depreciaciones (ver el apéndice).

El Cuadro 5 presenta el resumen estadístico de las principales variables utilizadas en nuestro análisis, clasificando las compañías según ventas anuales. Presentamos los datos para el periodo 1985-1996, los años posteriores a la crisis económica de 1982 y anteriores al impacto de la Crisis Asiática sobre la economía chilena. En el cuadro reportamos la variable de rentabilidad (Prof), la tasa de inversión (inversión como fracción del stock de capital rezagado) y el número de trabajadores. Esta última variable fue tabulada para 7 rangos de trabajadores, siendo 2 el rango más pequeño (10 a 19 trabajadores) y 8 el rango más grande (más de mil trabajadores). El número de observaciones por año en cada tipo de empresa (pequeña, mediana y grande) es bastante estable. Sin embargo, las compañías parecen diferir dentro de cada clasificación a través del tiempo. De hecho, las empresas pequeñas mostraron, en promedio, una rentabilidad de $0,77 \mathrm{y}$ un tasa de inversión igual a 1\% en 1985. Dichas variables aumentaron a 1,7 y 4\% en 1996, respectivamente. Un patrón similar sucede en el caso de las compañías medianas y grandes; las empresas medianas incrementaron su tasa de inversión de $1 \%$ en 1985 a 4\% en 1996, mientras que las empresas grandes lo hicieron desde un $2 \%$ en 1985 a un $6 \%$ en 1996.

El número promedio de trabajadores no varía considerablemente a través del tiempo entre las pequeñas y medianas empresas. Hay empresas pequeñas que tuvieron un número similar de empleados en 1985 y 1996 (aproximadamente 12 trabajadores). Similarmente, las compañías de tamaño medio tuvieron alrededor de 16 empleados en 1985 y 18 empleados en 1996. Sin embargo, el número de empleados aumentó en las compañías grandes desde un promedio de 56 trabajadores en 1985 hasta casi 82 trabajadores en 1996. Por ende, los datos sugieren un cambio en el patrón de inversión de las empresas, incluso considerando sus diferentes tamaños, mientras que el empleo parece estable a través del tiempo en las pequeñas y medianas empresas.

Nuestras estimaciones también incluyen variables agregadas de control, como manera de evaluar la importancia de shocks externos sobre las decisiones de inversión. Tales shocks pueden afectar la inversión específica a una empresa, no capturados por las variables explicativas discutidas previamente (también específicas a la empresa). Las variables explicativas comunes a todas las firmas son la brecha del producto (rezagada un año), los requerimientos legales de reservas mantenidas por los bancos privados con el Banco Central de Chile, la tasa de interés y la tasa de impuesto corporativo. La brecha del producto es la diferencia entre la tasa actual de crecimiento del PIB real y la tasa de crecimiento de tendencia 
del PIB (conocida también como producto potencial) 4 . Esta variable trata de controlar por el elemento cíclico del crecimiento económico y la inversión, y es usada en forma rezagada.

La variable de requerimientos de reservas (encaje bancario) trata de medir las restricciones globales de liquidez que enfrentan las empresas, las cuales, finalmente, determinan cuánta inversión se puede realizar en cada año. Los altos requerimientos de reservas se traducen en una menor liquidez y reducidas fuentes de financiamiento para las compañías que desean invertir.

Como una medida del costo relativo de la inversión, elegimos la tasa de interés real promedio de operaciones de 90 días a 1 año. Esta tasa representa el promedio de tasas de interés de 12 meses medidos entre mayo (año precedente) y abril (año corriente). Finalmente, la tasa de impuesto es la tasa de impuesto corporativo sobre las utilidades retenidas para las empresas chilenas. Se debe precisar que entre 1980 y 1986 la tasa de impuesto es el promedio del impuesto a las Sociedades Anónimas (SAs) y a las Sociedades de Responsabilidad Limitada (SRLs). Desde 1986, ambas tasas se igualaron. Las variables agregadas son presentadas en el Gráfico 1.

CUADRO 5

DESCRIPCION DE LAS EMPRESAS SEGUN TAMAÑO, ENIA 1985 Y 1996

\begin{tabular}{|c|c|c|c|c|c|c|c|}
\hline \multirow[b]{2}{*}{ Año } & \multicolumn{6}{|c|}{ Tamaño de las Empresas según Ventas Anuales } & \multirow[b]{2}{*}{ Max } \\
\hline & $\begin{array}{c}\text { Tamaño de las } \\
\text { empresas }\end{array}$ & Variable & Observaciones & Media & Desv. Est. & Min & \\
\hline \multirow{3}{*}{1985} & \multirow{3}{*}{ Pequeñas } & Prof & 152 & 0.77 & 0.85 & 0.02 & 4.25 \\
\hline & & $\operatorname{Inv} / \mathrm{k}(-1)$ & 152 & 0.01 & 0.03 & -0.14 & 0.29 \\
\hline & & Trabajadores & 152 & 2.27 & 0.45 & 2.00 & 3.00 \\
\hline \multirow{3}{*}{1985} & \multirow{3}{*}{ Medianas } & Prof & 289 & 0.74 & 0.78 & 0.01 & 4.15 \\
\hline & & $\operatorname{Inv} / \mathrm{k}(-1)$ & 289 & 0.01 & 0.09 & -0.22 & 1.24 \\
\hline & & Trabajadores & 289 & 2.65 & 0.55 & 2.00 & 4.00 \\
\hline \multirow{3}{*}{1985} & \multirow{3}{*}{ Grandes } & Prof & 511 & 0.60 & 0.67 & 0.01 & 4.63 \\
\hline & & $\operatorname{Inv} / \mathrm{k}(-1)$ & 511 & 0.02 & 0.09 & -0.36 & 1.15 \\
\hline & & Trabajadores & 511 & 4.13 & 1.30 & 2.00 & 8.00 \\
\hline \multirow{3}{*}{1996} & \multirow{3}{*}{ Pequeñas } & Prof & 136 & 1.70 & 1.24 & -0.48 & 4.92 \\
\hline & & $\operatorname{Inv} / \mathrm{k}(-1)$ & 136 & 0.04 & 0.14 & -0.24 & 1.04 \\
\hline & & Trabajadores & 136 & 2.24 & 0.43 & 2.00 & 3.00 \\
\hline \multirow{3}{*}{1996} & \multirow{3}{*}{ Medianas } & Prof & 259 & 1.77 & 1.18 & -0.37 & 4.83 \\
\hline & & Inv/k(-1) & 259 & 0.04 & 0.24 & -0.76 & 3.03 \\
\hline & & Trabajadores & 259 & 2.86 & 0.60 & 2.00 & 5.00 \\
\hline \multirow{3}{*}{1996} & \multirow{3}{*}{ Grandes } & Prof & 485 & 1.30 & 0.98 & -0.33 & 4.98 \\
\hline & & $\operatorname{Inv} / \mathrm{k}(-1)$ & 485 & 0.06 & 0.15 & -0.79 & 0.97 \\
\hline & & Trabajadores & 485 & 4.66 & 1.34 & 2.00 & 8.00 \\
\hline
\end{tabular}

Pequeñas y medianas empresas son aquellas con ventas anuales entre UF 2.400 y 25.000 (US\$ 72.500 a 754.000) y UF 25.000-100.000 (US\$ 754.000-3.000.000). Empresas grandes son aquellas con ventas anuales mayores a UF 100.000 (US\$ 3.000.000). La variable Inv/k(-1) es la tasa de inversión como fracción del stock de capital rezagado mientras que la variable trabajadores indica el número de trabajadores empleados por empresa. Esta variable toma valores dentro de un rango que va de 2 a 8 , donde 2 indica empresas con 10 a 19 trabajadores; 3 indica 20 a 49; 4 indica 50 a 99; 5 indica 100 a 199; 6 indica 200 a 499; 7 indica 500 a 999 y 8 indica más de 1.000 trabajadores por empresa.

${ }^{4}$ El producto potencial se calcula usando el filtro de Hodrick-Precott. 


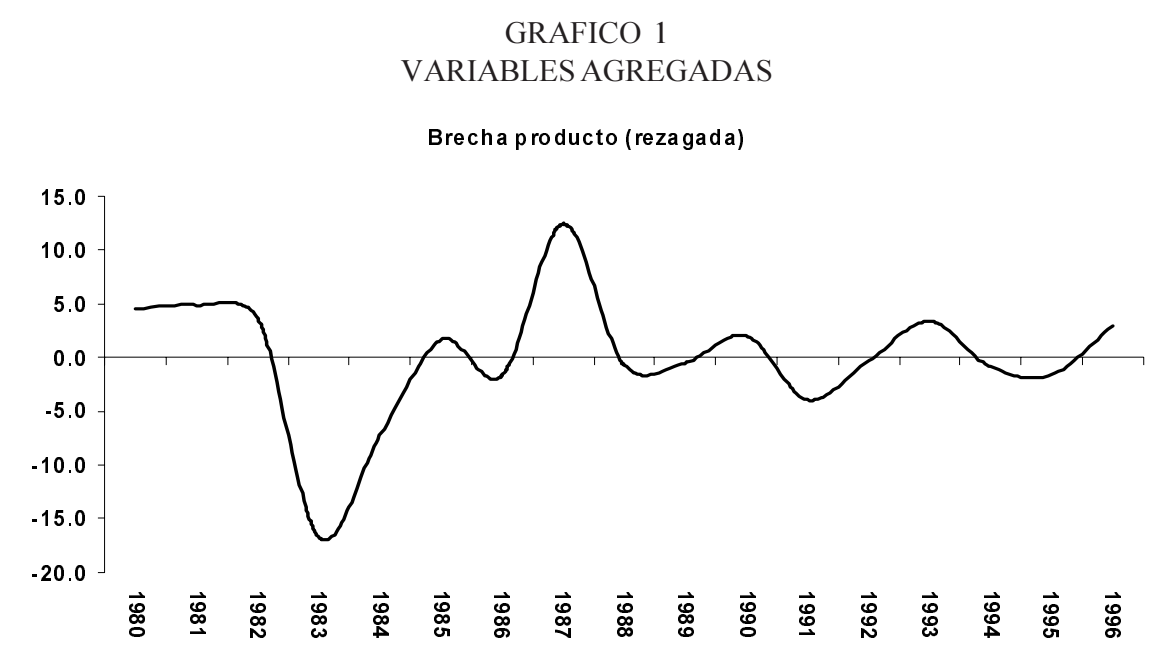

Requerimientos de Reservas de los Bancos Privados

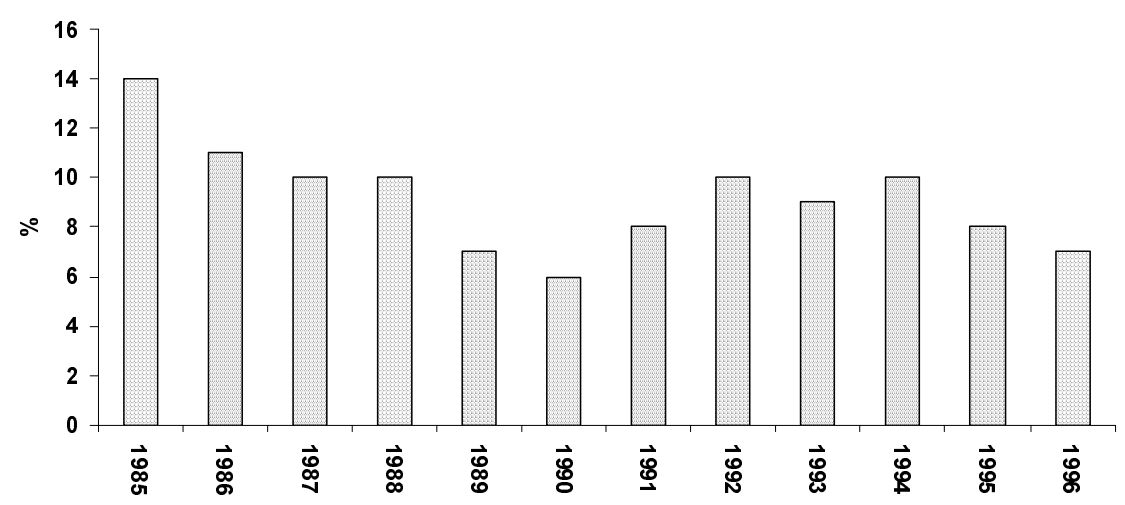

Tasa de Interés

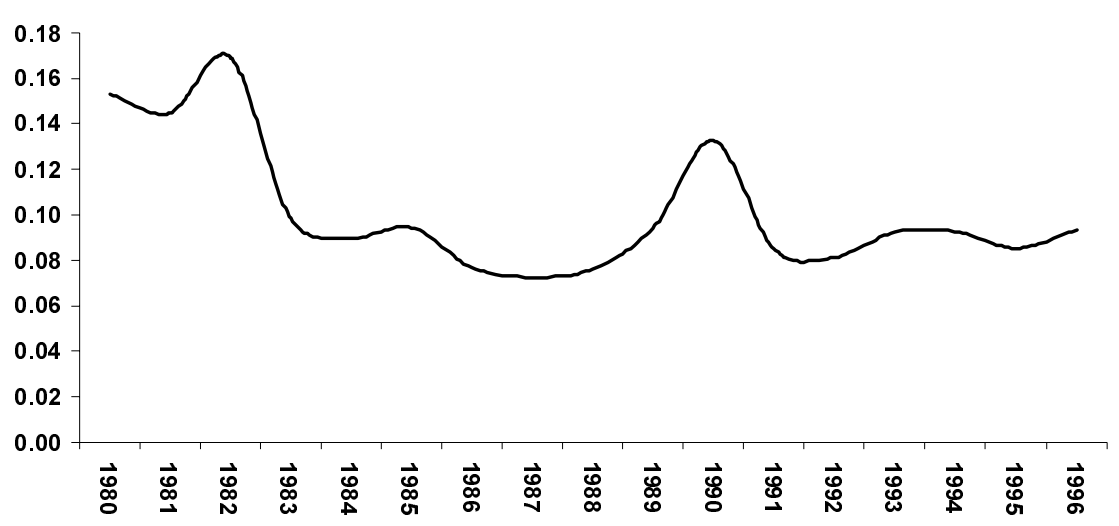




\author{
CONT. GRAFICO 1
}

Tasa de Impuesto

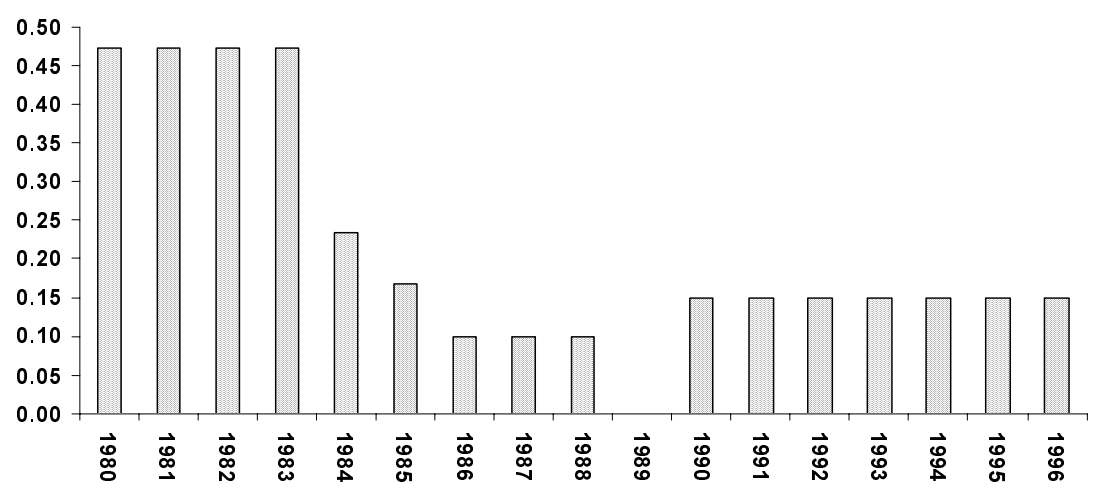

La tasa de impuesto corporativo sobre las utilidades retenidas de las empresas chilenas ha sido modificada (generalmente reducida) muchas veces producto de las reformas tributarias en la economía chilena durante los años ochenta y noventa. Al comienzo de nuestra muestra, la tasa de impuesto fue $46 \%$ para las SAs y $48 \%$ para las SRLs. Ambas tasas fueron reducidas gradualmente, llegando, finalmente, ambas a $10 \%$ en 1986 . Las mayores variaciones de esta tasa ocurrieron durante 1989, cuando la tasa se fijó en 0 y, nuevamente, en 1990, cuando la tasa sobre las utilidades retenidas fue fijada en $15 \%$.

\title{
4. Resultados
}

En esta sección presentamos y discutimos los principales resultados. Nos centramos en dos tipos diferentes de estimaciones. Primero presentamos los resultados de estimaciones con efectos fijos; luego ofrecemos la evidencia arrojada de la estimación de paneles dinámicos de acuerdo a la metodología de Arellano-Bond. Finalmente consideramos cómo las estimaciones con datos micro difieren entre empresas de distinto tamaño.

El Cuadro 6 muestra los resultados usando las metodologías anteriormente señaladas. Las columnas 1 a 3 del cuadro presentan las estimaciones de efectos fijos, mientras que las columnas siguientes muestran los resultados derivados de las estimaciones de paneles dinámicos.

La primera columna del Cuadro 6 muestra la ecuación base en la cual incluimos la tasa de interés, la variable de rentabilidad y la tasa de impuestos corporativos. La segunda columna presenta los resultados de la ecuación base extendida para considerar variables adicionales que controlan por los shocks agregados a la economía que pueden afectar las decisiones de inversión más allá de los determinantes incluidos en la columna 1 del Cuadro 6. En esta columna (2), introducimos la brecha producto y los requerimientos de reserva como variables explicativas. 
Finalmente, la columna 3 del cuadro presenta las estimaciones con efectos fijos, incluyendo variables dummies por sector económico. Las columnas 4 a 6 incluyen estimaciones usando la metodología de Arellano-Bond. Los test de las regresiones Arellano-Bond muestran que se acepta el test de Sargan sobre las condiciones de ortogonalidad (columnas 5 y 6 ), mientras que se rechaza el test de correlación serial AR(2). ${ }^{5}$

Como podía esperarse, y contradiciendo la evidencia previa para la economía chilena, nuestros resultados muestran un fuerte y robusto efecto negativo de las tasas de impuesto sobre la tasa total de inversión, tal como se ve en el coeficiente de la tasa de impuesto corporativo en las diferentes especificaciones econométricas.

\begin{tabular}{|c|c|c|c|c|c|c|}
\hline & $\begin{array}{l}\text { Método } \\
\text { EF }\end{array}$ & $\begin{array}{l}\text { Método } \\
\text { EF }\end{array}$ & $\begin{array}{l}\text { Método } \\
\text { EF }\end{array}$ & $\begin{array}{l}\text { Método } \\
\text { AB }\end{array}$ & $\begin{array}{l}\text { Método } \\
\text { AB }\end{array}$ & $\begin{array}{l}\text { Método } \\
\mathrm{AB}\end{array}$ \\
\hline Tasa de interés & $\begin{array}{l}-0.032 \\
(-0.78)\end{array}$ & $\begin{array}{c}-0.10 \\
(-1.34)\end{array}$ & $\begin{array}{c}-0.10 \\
(-1.31)\end{array}$ & $\begin{array}{c}0.10 \\
(2.09)\end{array}$ & $\begin{array}{c}-0.19 \\
(-2.18)\end{array}$ & $\begin{array}{c}-0.19 \\
(-2.21)\end{array}$ \\
\hline Rentabilidad & $\begin{array}{l}0.029 \\
(21.8)\end{array}$ & $\begin{array}{l}0.031 \\
(16.2)\end{array}$ & $\begin{array}{l}0.031 \\
(16.2)\end{array}$ & $\begin{array}{l}0.06 \\
(26.2)\end{array}$ & $\begin{array}{l}0.07 \\
(26.5)\end{array}$ & $\begin{array}{c}0.07 \\
(26.4)\end{array}$ \\
\hline Impuesto & $\begin{array}{l}-0.02 \\
(-2.70)\end{array}$ & $\begin{array}{l}-0.039 \\
(-3.72)\end{array}$ & $\begin{array}{l}-0.039 \\
(-3.75)\end{array}$ & $\begin{array}{l}-0.09 \\
(-9.79)\end{array}$ & $\begin{array}{l}-0.10 \\
(-8.05)\end{array}$ & $\begin{array}{l}-0.10 \\
(-8.00)\end{array}$ \\
\hline Brecha producto & & $\begin{array}{l}-0.000 \\
(-0.60)\end{array}$ & $\begin{array}{l}-0.000 \\
(-0.56)\end{array}$ & & $\begin{array}{l}-0.000 \\
(-1.03)\end{array}$ & $\begin{array}{l}-0.000 \\
(-1.01)\end{array}$ \\
\hline Req. reservas & & $\begin{array}{l}-0.002 \\
(-4.00)\end{array}$ & $\begin{array}{l}-0.002 \\
(-3.95)\end{array}$ & & $\begin{array}{l}-0.003 \\
(-5.94)\end{array}$ & $\begin{array}{l}-0.003 \\
(-5.89)\end{array}$ \\
\hline $\begin{array}{l}\text { Dummies por sector } \\
\text { económico }\end{array}$ & No & No & $\mathrm{Si}$ & No & No & $\mathrm{Si}$ \\
\hline $\mathrm{R}^{2}$ & 0.030 & 0.021 & 0.016 & & & \\
\hline $\mathrm{R}^{2}$ within & 0.037 & 0.030 & 0.031 & & & \\
\hline $\mathrm{R}^{2}$ between & 0.027 & 0.008 & 0.004 & & & \\
\hline Grupos & 967 & 964 & 964 & 967 & 963 & 963 \\
\hline Observaciones & 14900 & 11060 & 11060 & 13003 & 10142 & 10142 \\
\hline Rezago I/k(-1) & & & & 1 & 1 & 1 \\
\hline Test de Sargan & & & & $\begin{array}{l}534.9 \\
(0.00)\end{array}$ & $\begin{array}{l}401.9 \\
(0.99)\end{array}$ & $\begin{array}{l}400.1 \\
(0.99)\end{array}$ \\
\hline $\begin{array}{l}\text { Autocorrelación } \\
\text { orden } 1\end{array}$ & & & & $\begin{array}{l}-53.3 \\
(0.00)\end{array}$ & $\begin{array}{l}-45.5 \\
(0.00)\end{array}$ & $\begin{array}{l}-45.3 \\
(0.00)\end{array}$ \\
\hline $\begin{array}{l}\text { Autocorrelación } \\
\text { orden } 2\end{array}$ & & & & $\begin{array}{l}-0.99 \\
(0.32)\end{array}$ & $\begin{array}{l}-1.65 \\
(0.11)\end{array}$ & $\begin{array}{l}-1.63 \\
(0.11)\end{array}$ \\
\hline
\end{tabular}

Nota: Estadístico $\mathrm{Z}$ en paréntesis. Las constantes fueron estimadas, pero no mostradas en el cuadro.

\footnotetext{
${ }^{5}$ En el contexto de regresiones Arellano-Bond GMM, las cuales son estimadas en primeras diferencias, se espera un $\mathrm{AR}(1)$. El test $\mathrm{AR}(2)$ sobre los residuos en primeras diferencias es usado para detectar $\mathrm{AR}(1)$ en los niveles de las variables.
} 


\section{Discriminando por el tamaño de las empresas}

A continuación, analizaremos cómo cambian los diferentes parámetros cuando elegimos diferentes submuestras de acuerdo al tamaño de las empresas. Clasificaremos los diferentes tamaños de las compañías de acuerdo a su volumen de ventas durante un año específico. En el Cuadro 7 presentamos las estimaciones por quintil de tamaño de las compañías. El panel (a) del Cuadro 7 presenta estimaciones usando efectos fijos, mientras que en el panel (b) del Cuadro 7 muestra estimaciones utilizando paneles dinámicos.

La primera columna de ambos cuadros representa el quintil de compañías con menores ventas, mientras que la quinta columna representa el quintil de empresas de mayor tamaño. Los resultados muestran que el impacto de la tasa de impuestos es negativo en todos los quintiles. Además, la magnitud del impacto de la tasa de impuesto es grande en los dos primeros quintiles y decrece establemente desde el tercer al quinto quintil. Más interesante aún, el impacto en las dos primeras columnas es altamente significativo, mientras que no lo es en el tercer y quinto quintil. Nótese el hecho de que las compañías del quinto quintil tienen ventas anuales mayores a un millón de UF (US\$ 25 millones), las cuales constituyen un conjunto de empresas similares a las analizadas por Bustos et al. (2004).

Por consiguiente, nuestras estimaciones sugieren que el impacto de la tasa de impuesto corporativo sobre las decisiones de inversión de una empresa es negativo, tal como lo sugiere la teoría económica; adicionalmente, la evidencia muestra que el impacto es de menor magnitud en las compañías grandes. El tamaño de una empresa parece ser, entonces, un elemento crucial al momento de considerar el impacto de los impuestos. Nuestra interpretación es que las empresas pequeñas y medianas generalmente enfrentan restricciones crediticias y necesitan utilizar fondos generados internamente para financiar su inversión, lo cual parece ser menos importante en compañías grandes que pueden endeudarse en los mercados financieros. Esto podría explicar los resultados reportados por Bustos et al. (2004) para Chile. Otros resultados presentados en el cuadro son: (1) el efecto de la rentabilidad sobre la inversión de las empresas es estable entre firmas de distinto tamaño; (2) el rezago de la variable dependiente es usualmente no significativo; (3) el impacto del ciclo económico es también importante para las empresas pequeñas, y (4) el impacto de la tasa de interés es generalmente negativo, pero no significativo. 


\section{CUADRO 7}

RESULTADOS SEGUN TAMAÑO DE LA EMPRESAS

(estimaciones de paneles dinámicos Arellano-Bond)

(a) Método de Efectos Fijos

\begin{tabular}{|c|c|c|c|c|c|}
\hline \multicolumn{6}{|c|}{ Ecuaciones según tamaño de las empresas (agregado por quintiles), ecuación con variables de control } \\
\hline & Q1 & Q2 & Q3 & Q4 & Q5 \\
\hline \multirow[t]{2}{*}{ Impuesto } & -0.05 & -0.087 & -0.03 & -0.05 & 0.01 \\
\hline & $(-2.45)$ & $(-4.02)$ & $(-1.10)$ & $(-2.11)$ & $(0.49)$ \\
\hline \multirow[t]{2}{*}{ Tasa de interés } & 0.06 & -0.11 & -0.11 & -0.15 & -0.14 \\
\hline & $(0.40)$ & $(-0.75)$ & $(-0.54)$ & $(-0.82)$ & $(-0.86)$ \\
\hline \multirow[t]{2}{*}{ Rentabilidad } & 0.020 & 0.016 & 0.042 & 0.047 & 0.046 \\
\hline & $(5.83)$ & $(4.52)$ & $(8.45)$ & $(9.29)$ & $(9.53)$ \\
\hline \multirow[t]{2}{*}{ Brecha producto } & 0.001 & -0.000 & 0.000 & -0.000 & -0.000 \\
\hline & $(2.27)$ & $(-1.54)$ & $(0.14)$ & $(-0.52)$ & $(-1.15)$ \\
\hline \multirow[t]{2}{*}{ Req. reservas } & -0.002 & -0.001 & -0.002 & -0.001 & -0.002 \\
\hline & $(-1.86)$ & $(-1.70)$ & $(-2.02)$ & $(-0.79)$ & $(-1.78)$ \\
\hline $\mathrm{R}^{2}$ & 0.01 & 0.01 & 0.02 & 0.02 & 0.05 \\
\hline $\mathrm{R}^{2}$ within & 0.02 & 0.02 & 0.04 & 0.05 & 0.06 \\
\hline $\mathrm{R}^{2}$ between & 0.002 & 0.001 & 0.01 & 0.02 & 0.07 \\
\hline No. de obs. & 2137 & 2158 & 2233 & 2299 & 2263 \\
\hline No. de empresas & 191 & 191 & 193 & 195 & 194 \\
\hline
\end{tabular}

Nota: Estadístico $\mathrm{Z}$ en paréntesis. Las constantes fueron estimadas, pero no mostradas en el cuadro.

(b) Paneles Dinámicos Arellano-Bond

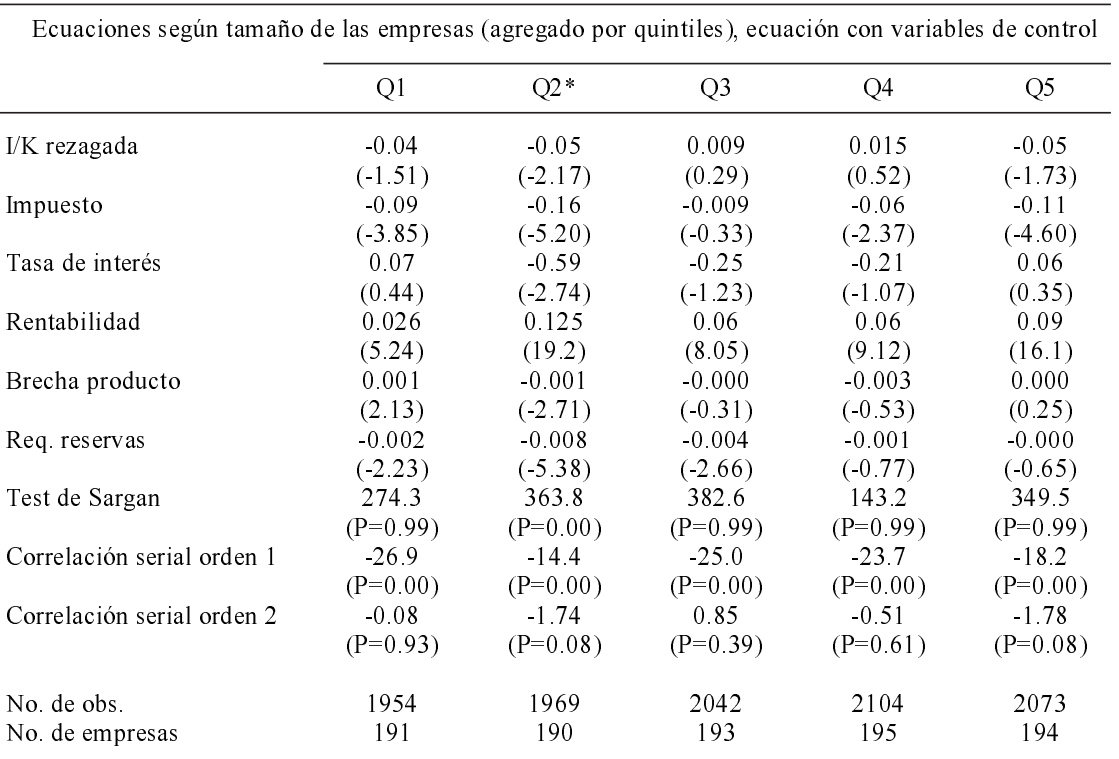

Nota: incluye dos rezagos de la variable dependiente no aportados.

Estadístico $\mathrm{Z}$ en paréntesis. Las constantes fueron estimadas, pero no mostradas en el cuadro. 
A modo de realizar un análisis de sensibilidad de nuestros resultados, estimamos regresiones móviles basadas en el nivel de ventas reales de las empresas - una variable continua- la cual podemos usar para calcular los efectos para distintos tamaños de empresas. El Gráfico 2 presenta los resultados para el coeficiente ligado a la variable de impuesto bajo diferentes regresiones y estimado utilizando muestras móviles en que se hace variar el volumen de ventas reales. El Panel (a) del Gráfico 2, muestra el coeficiente de la variable de impuesto de la ecuación extendida usando la metodología de efectos fijos (tercera columna en el Cuadro 6), estimada para diferentes muestras. Dichas muestras móviles fueron elegidas específicamente para mantener un tamaño muestral similar en cada regresión. Por tanto, en cada una de las estimaciones recursivas fue considerado un promedio de 250 empresas. El Panel (b) muestra las estimaciones alternativas en las cuales fue utilizada la metodología de Arellano-Bond (sexta columna en el Cuadro 6).

Puesto que las pequeñas y medianas empresas en Chile se definen de acuerdo a su nivel de ventas anuales, los Gráficos 2 a y $2 b$ muestran directamente si el efecto de las tasas de impuesto sobre la inversión es disímil entre empresas de distinto tamaño. El gráfico muestra claramente que existe un punto de corte a partir del cual el efecto de la tasa de impuesto decrece considerablemente. El punto de corte ocurre aproximadamente en la regresión \#320, la cual coincide con la 570 empresa más pequeña de la muestra (percentil 60\%). Dicha empresa, en promedio, vende UF 200.000 por año (aproximadamente, US\$6 MM). Por consiguiente, la evidencia confirma la intuición de que la inversión es afectada más significativamente por cambios en las tasas de impuesto en las empresas pequeñas que en las empresas grandes. Una conclusión similar se desprende del panel (b), que presenta estimaciones con la metodología de Arellano-Bond. Ambos paneles difieren en dos características: (1) el impacto de la tasa de impuesto corporativo es mayor usando la metodología de Arellano-Bond, y (2) el 10\% de las firmas más grandes presenta un impacto negativo de los impuestos sólo en el segundo panel. 


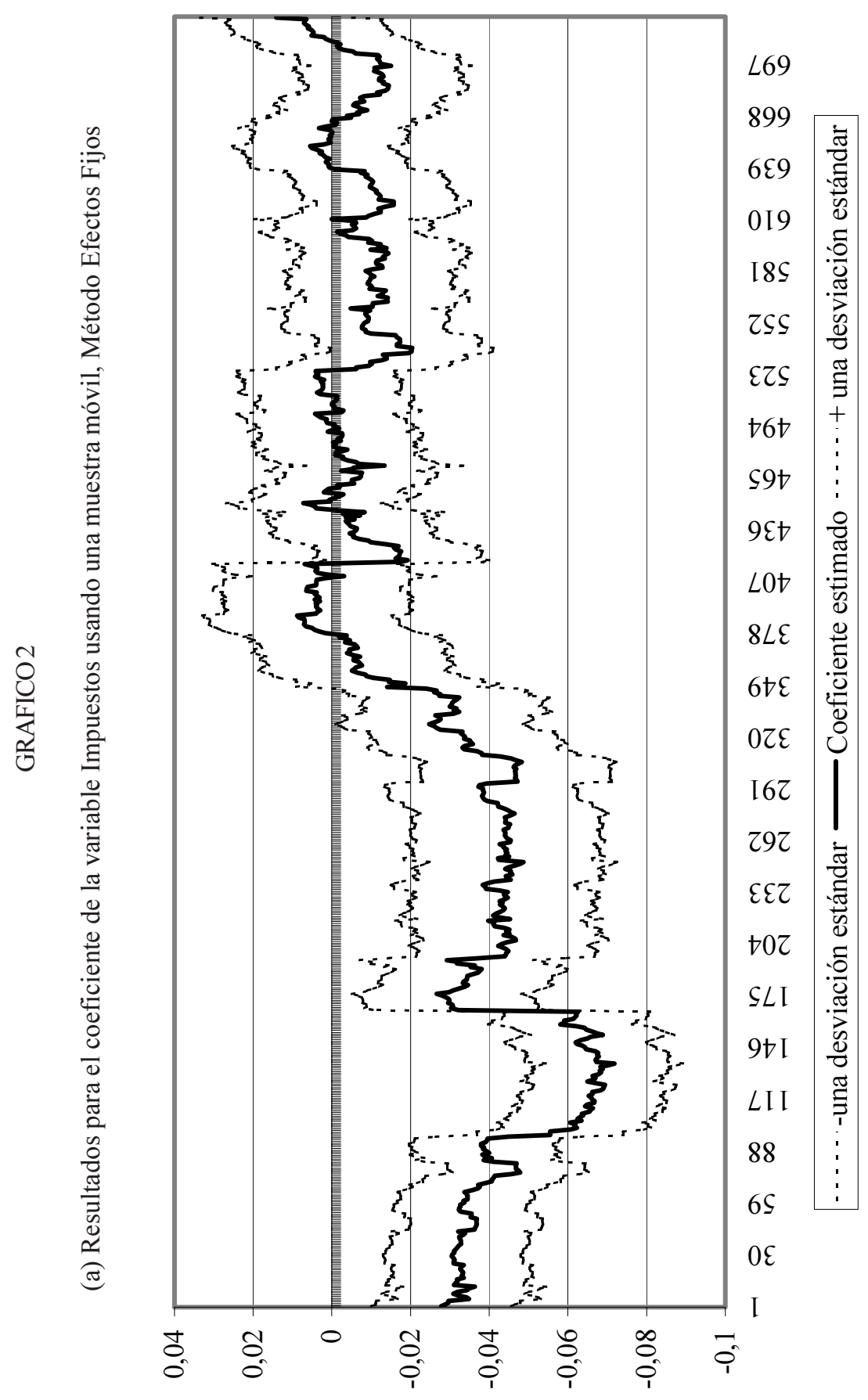




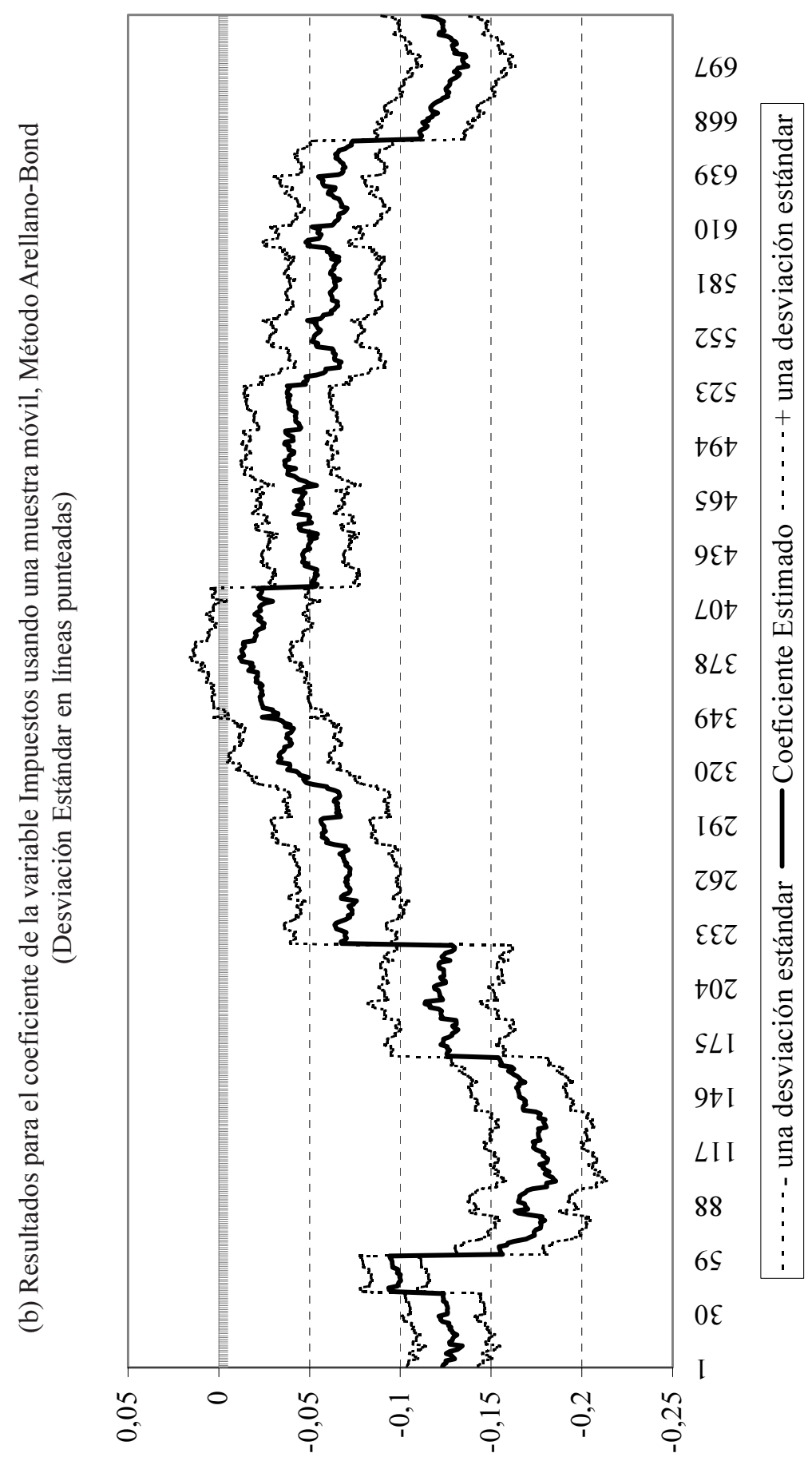




\section{Restricciones crediticias y tamaño de las empresas}

Hasta ahora, hemos proporcionado evidencia indicando que la tasa de impuestos corporativos tiene un impacto diferente según el tamaño de la empresa. Hemos interpretado este resultado como un potencial efecto de las restricciones crediticias sobre las pequeñas empresas, las cuales deben financiar su inversión usando preferentemente flujos de caja internos en vez de endeudarse en el mercado de capitales. Sin embargo, no hemos proporcionado aún evidencia de esta conjetura. En esta sección nos enfocamos precisamente en la evidencia que la soporta.

Nuestra idea inicial fue incluir una medida de restricciones crediticias en nuestras regresiones como una variable de control adicional. Lamentablemente, nuestros datos no incluyen una medida directa de flujo de caja o restricciones de crédito. Con el fin de proporcionar evidencia del impacto de las restricciones crediticias sobre la inversión, seguimos un enfoque alternativo. En vez de usar datos microeconómicos, buscamos una variable macro que mida el desarrollo del sector financiero en Chile. Para ello, obtenemos de Díaz, Lüders y Wagner (2005) una serie llamada FIR (Financial Intermediation Ratio), la cual se mide como la suma de los depósitos bancarios, la deuda hipotecaria y el valor de las acciones como porcentaje del PIB para cada año de nuestra muestra. El Gráfico 3 muestra la evolución de esta variable entre 1960 y 2003 . Tal como puede ser visto en el gráfico, esta variable muestra una varianza significativa, especialmente en el subperiodo de nuestra muestra.

GRAFICO 3

INTERMEDIACION FINANCIERA (\% PIB) CHILE 1960-2000

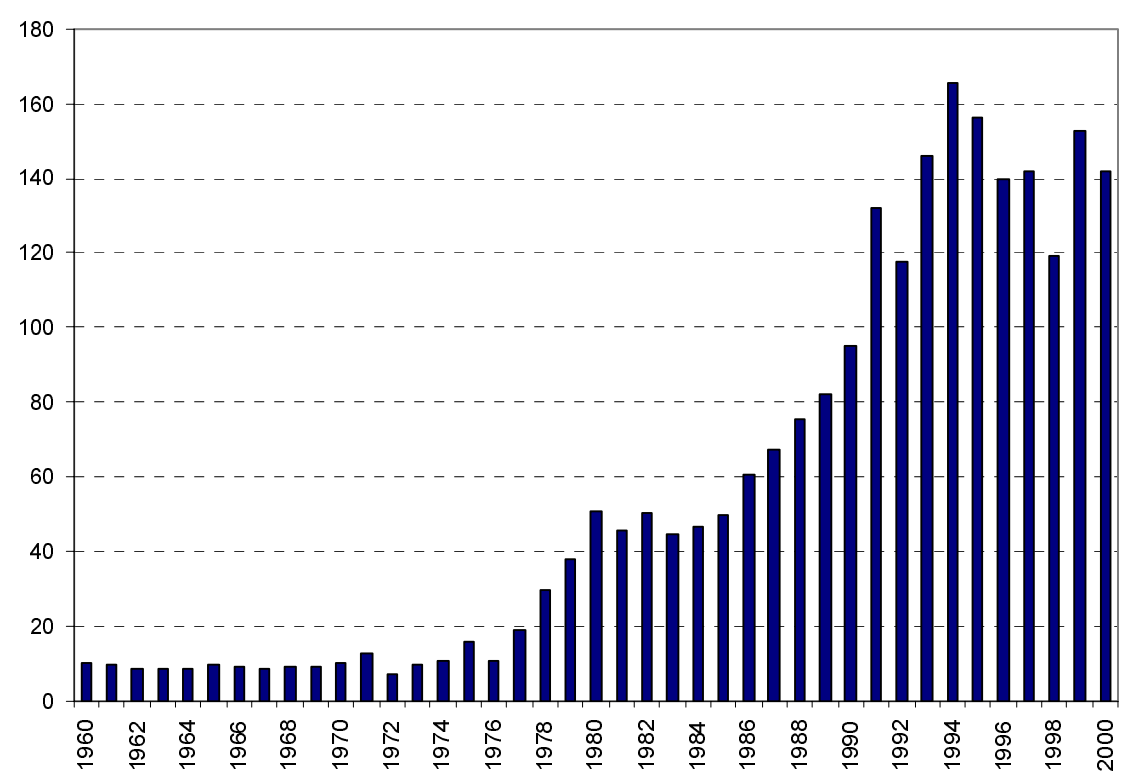


Incluiremos la variable FIR y permitiremos que ella interactúe con la tasa de impuesto. La idea de incluir esta interacción es que mientras mayor es el desarrollo del sector financiero, más probable es la existencia de una gran disponibilidad de crédito para las firmas; por tanto, menor debiese ser el impacto de la tasa de impuesto sobre la inversión, si es que la causa de la asimetría del impacto de la tasa de impuesto es la existencia de restricciones crediticias. Así, estimamos la siguiente regresión:

$$
\left(\frac{I}{K_{-1}}\right)_{i t}=\beta_{0} \operatorname{Prof}_{i t}+\beta_{1} i_{t}+\beta_{2} \operatorname{Tax}_{t}+\beta_{3} \operatorname{Tax}_{t} * \operatorname{FIR}_{t}+\theta X_{t}+\mu_{i}+\varepsilon_{i t}
$$

donde $\mathrm{X}$ denota un conjunto de variables de control que incluye la brecha producto, los requerimientos de reservas y variables dicotómicas por sectores económicos, mientras que $\mu_{i}$ es un efecto fijo. El Cuadro 8 presenta los resultados derivados de las estimaciones de efectos fijos y paneles dinámicos Arellano-Bond para la muestra completa.

CUADRO 8

RESULTADOS DE ESTIMACIONES CON EFECTOS FIJOS (EF) Y PANELES DINAMICOS ARELLANO BOND (AB)

\begin{tabular}{|c|c|c|c|c|c|c|}
\hline & $\begin{array}{l}\text { Método } \\
\text { EF }\end{array}$ & $\begin{array}{l}\text { Método } \\
\text { EF }\end{array}$ & $\begin{array}{l}\text { Método } \\
\text { EF }\end{array}$ & $\begin{array}{l}\text { Método } \\
\text { AB }\end{array}$ & $\begin{array}{l}\text { Método } \\
\text { AB }\end{array}$ & $\begin{array}{l}\text { Método } \\
\text { AB }\end{array}$ \\
\hline Tasa de interés & $\begin{array}{l}0.029 \\
(0.75)\end{array}$ & $\begin{array}{l}0.024 \\
(0.26)\end{array}$ & $\begin{array}{l}0.024 \\
(0.26)\end{array}$ & $\begin{array}{c}-0.22 \\
(-4.17)\end{array}$ & $\begin{array}{c}-0.18 \\
(-1.51)\end{array}$ & $\begin{array}{c}-0.18 \\
(-1.61)\end{array}$ \\
\hline Rentabilidad & $\begin{array}{l}0.023 \\
(14.3)\end{array}$ & $\begin{array}{l}0.028 \\
(13.4)\end{array}$ & $\begin{array}{l}0.028 \\
(13.5)\end{array}$ & $\begin{array}{c}0.06 \\
(26.7)\end{array}$ & $\begin{array}{c}0.07 \\
(26.7)\end{array}$ & $\begin{array}{c}0.07 \\
(26.6)\end{array}$ \\
\hline Impuesto & $\begin{array}{l}-0.062 \\
(-6.81)\end{array}$ & $\begin{array}{c}-0.16 \\
(-3.32)\end{array}$ & $\begin{array}{c}-0.16 \\
(-3.26)\end{array}$ & $\begin{array}{l}-0.12 \\
(-1.94)\end{array}$ & $\begin{array}{l}-2.47 \\
(-3.68)\end{array}$ & $\begin{array}{l}-2.50 \\
(-3.71)\end{array}$ \\
\hline Impuesto*FIR & $\begin{array}{c}0.0006 \\
(4.46)\end{array}$ & $\begin{array}{c}0.0007 \\
(2.80)\end{array}$ & $\begin{array}{c}0.0007 \\
(2.73)\end{array}$ & $\begin{array}{l}0.001 \\
(1.53)\end{array}$ & $\begin{array}{l}0.050 \\
(3.58)\end{array}$ & $\begin{array}{l}0.051 \\
(3.62)\end{array}$ \\
\hline Brecha producto & & $\begin{array}{c}-0.0003 \\
(-1.12)\end{array}$ & $\begin{array}{c}-0.0003 \\
(-1.09)\end{array}$ & & $\begin{array}{l}0.001 \\
(2.43)\end{array}$ & $\begin{array}{l}0.001 \\
(2.49)\end{array}$ \\
\hline Req. reservas & & $\begin{array}{c}0.0004 \\
(0.52)\end{array}$ & $\begin{array}{c}0.0004 \\
(0.52)\end{array}$ & & $\begin{array}{l}-0.001 \\
(-0.83)\end{array}$ & $\begin{array}{l}-0.001 \\
(-0.30)\end{array}$ \\
\hline $\begin{array}{l}\text { Dummies por sector } \\
\text { económico }\end{array}$ & No & No & Yes & No & No & Yes \\
\hline $\begin{array}{l}\mathrm{R}^{2} \\
\mathrm{R}^{2} \text { within } \\
\mathrm{R}^{2} \text { between }\end{array}$ & $\begin{array}{l}0.04 \\
0.04 \\
0.03\end{array}$ & $\begin{array}{l}0.02 \\
0.03 \\
0.01\end{array}$ & $\begin{array}{l}0.02 \\
0.03 \\
0.01\end{array}$ & & & \\
\hline $\begin{array}{l}\text { Grupos } \\
\text { Observacions }\end{array}$ & $\begin{array}{c}967 \\
14901\end{array}$ & $\begin{array}{c}964 \\
11091\end{array}$ & $\begin{array}{c}964 \\
11091\end{array}$ & $\begin{array}{c}963 \\
10143\end{array}$ & $\begin{array}{c}963 \\
10143\end{array}$ & $\begin{array}{c}963 \\
10143\end{array}$ \\
\hline Rezago I/k(-1) & & & & $\begin{array}{l}0.001 \\
(0.13)\end{array}$ & $\begin{array}{l}0.003 \\
(0.25)\end{array}$ & $\begin{array}{l}0.004 \\
(0.31)\end{array}$ \\
\hline Test de Sargan & & & & $\begin{array}{c}527.2 \\
(\mathrm{P}=0.99)\end{array}$ & $\begin{array}{c}394.1 \\
(\mathrm{P}=0.99)\end{array}$ & $\begin{array}{c}393.3 \\
(\mathrm{P}=0.99)\end{array}$ \\
\hline Autocorrelación orden 1 & & & & $\begin{array}{c}-53.2 \\
(\mathrm{P}=0.00)\end{array}$ & $\begin{array}{c}-45.5 \\
(\mathrm{P}=0.00)\end{array}$ & $\begin{array}{c}-45.4 \\
(\mathrm{P}=0.00)\end{array}$ \\
\hline Autocorrelación orden 2 & & & & $\begin{array}{c}-1.16 \\
(\mathrm{P}=0.24)\end{array}$ & $\begin{array}{c}-1.39 \\
(\mathrm{P}=0.16)\end{array}$ & $\begin{array}{c}-1.37 \\
(\mathrm{Pp}=0.17)\end{array}$ \\
\hline
\end{tabular}

Nota:Estadístico $\mathrm{Z}$ en paréntesis. Las constantes fueron estimadas, pero no mostradas en el cuadro. 
Los resultados exhibidos en el Cuadro 8 son similares a los que obtuvimos previamente: el efecto de la rentabilidad en la inversión es positivo y altamente significativo, el de la tasa de interés es generalmente negativo, pero no significativo y el de la tasa de impuesto es negativo y significativo. El término de interacción es positivo, indicando que mientras mayor es la disponibilidad de crédito para las empresas, menor es el impacto de la tasa de impuesto corporativo sobre la inversión. Nótese que la estimación del impacto de la tasa de impuestos sobre la inversión es $\beta_{2}+\beta_{3} F I R_{t}$. El Gráfico 4 muestra la evolución de este coeficiente en el tiempo. Tal como se muestra en el gráfico, al principio de la muestra, en promedio, el impacto de la tasa de impuesto corporativo sobre la tasa de inversión fue tres veces mayor al que tuvo al final de la muestra. Los resultados indican que un incremento de $10 \%$ en la tasa de impuesto corporativo debería disminuir la inversión como fracción del stock de capital en un 1,2\% en 1980, pero sólo en un $0,06 \%$ en 1996.

GRAFICO 4

IMPACTO DE LA TASA DE IMPUESTO CORPORATIVO SOBRE LA INVERSION, CHILE 1980-2000

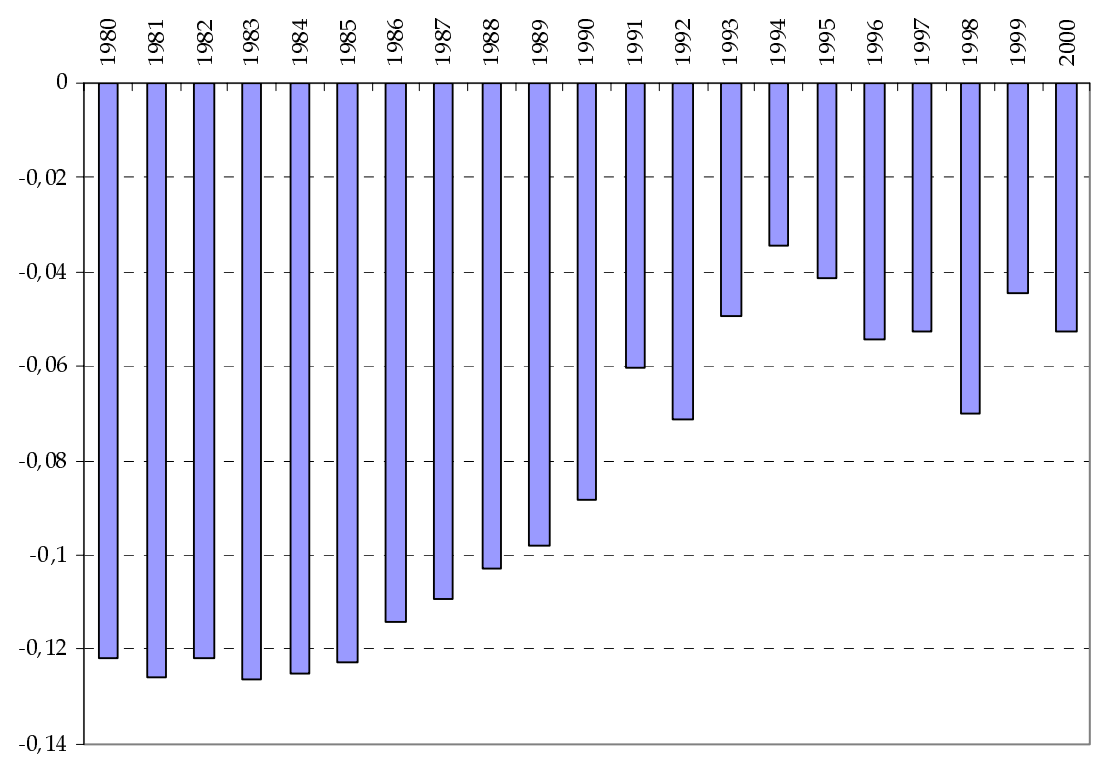

\section{Conclusiones}

Este trabajo proporciona evidencia empírica acerca del impacto de la tasa de impuesto corporativo sobre las decisiones de inversión de las empresas en Chile. Analizar este caso es sumamente interesante, dados los considerables cam- 
bios en las tasas de impuesto corporativos durante el periodo de análisis. Adicionalmente, nuestros datos nos permiten distinguir entre tipos de empresas. En nuestras estimaciones, encontramos diferencias significativas en el impacto de la tasa de impuesto corporativo según el tamaño de la firma, lo cual sugiere la existencia de restricciones crediticias en empresas de menor tamaño.

La evidencia microeconómica muestra un impacto negativo y significativo de la tasa de impuesto corporativo sobre la inversión: un incremento de $10 \%$ de la tasa de impuesto reduce la inversión como fracción del stock de capital entre $0,2 \%$ y $1 \%$ bajo diferentes especificaciones econométricas. Dicho impacto difiere entre empresas de distinto tamaño. En pequeñas y medianas empresas, la inversión como fracción del stock de capital decrece entre $0,5 \%$ y 1,6\%, mientras que en compañías grandes el impacto es negativo pero no significativo. Adicionalmente, si controlamos por una medida del desarrollo financiero de la economía, los efectos de los tributación corporativa sobre la inversión son mayores: un incremento de $10 \%$ en la tasa de impuesto corporativo disminuye la inversión como fracción del stock de capital entre $0,6 \%$ y 2,5\%. Además, nuestra evidencia sugiere que mientras mayor es la disponibilidad de crédito, menor es el impacto de la tasa de impuesto corporativo sobre la inversión.

Estos resultados proveen soporte a la visión de que la tasa de impuesto corporativo es un determinante clave de la inversión privada, especialmente en países en desarrollo -donde las restricciones crediticias son más importantes que en el caso de los países desarollados-y especialmente para las pequeñas y medianas empresas, las cuales enfrentan más restricciones crediticias que las compañías grandes $\mathrm{y}$, por consiguiente, dependen fuertemente de las utilidades retenidas para financiar inversión.

\section{REFERENCIAS}

Aguilar, X. y M.P., Collinao (2001), “Cálculo del Stock de Capital para Chile 1985-2000”, Documento de Trabajo 133, Banco Central de Chile.

Arellano, M. y S. Bond (1991), "Some Test of Specification for Panel Data: Montecarlo Evidence and An Application to Employment Equations", Review of Economic Studies, 58: 277-297.

Bustos, A.; E., Engel, y A. Galetovic (2004), "Could Higher Taxes Increase the Long-run Demand for Capital? Theory and Evidence for Chile", Journal of Development Economics, 73: 675-697.

Cabrera, A.; S. de la Cuadra, A. Galetovic y R. Sanhueza (2002), "Las Pyme: ¿Quiénes son?, ¿Cómo son y qué hacer con ellas?”, mimeo, Sociedad de Fomento Fabril, Chile.

Coeymans, J.E. (2001), "Productividad y Desempleo: Consideraciones para un Análisis Prospectivo", mimeo, Mideplan, Chile.

Cummins, J.; K., Hassett y G., Hubbard (1994), “A Reconsideration of Investment Behavior Using Tax Reforms as Natural Experiments", Brookings Papers in Economic Activity, 2: 1-59. 
Díaz, J.; R. Lüders y G. Wagner (2005), La República en Cifras: Chile, 1810-2000. Por aparecer, Santiago, Banco Central de Chile.

Du Toit, Ch. y E. Moolman (2004), “A Neoclassical Investment Function of the South African Economy", Economic Modelling, 21(4): 647-660.

Fazzari S.; G. Hubbard y M. Petersen (1988), "Financing Constraints and Corporate Investment," Brooking Papers on Economic Activity, 1:141-95.

Gutiérrez, M. (1987), “Ahorro Interno y Crecimiento Económico: Un Enfoque de Cuentas Nacionales", Cuadernos de Economía, 73: 331-357.

Hall, R. y D. Jorgenson (1967), “Tax Policy and Investment Behavior", American Economic Review, 57: 391- 414.

Hsieh, C. y J. Parker (2002), "Taxes and Growth in a Financially Underdeveloped Country: Evidence from the Chilean Investment Boom", mimeo, Princeton University.

Hubbard, G.; A. K. Kashyap y T. Whited (1995), "Internal Finance and Firm Investment", Journal of Money, Credit and Banking, 27: 683-701.

Jorgenson, D. (1963), “Capital Theory Investment Behavior", American Economic Review Papers and Proceedings, 53: 247-259.

Medina, J.P. y R. Valdés (1998). "Flujo de Caja y Decisiones de Inversión en Chile: Evidencia de Sociedades Anónimas Abiertas", Cuadernos de Economía, 106: 301-323.

Modigliani, F. y M.H. Miller (1958), "The Cost of Capital, Corporate Finance, and the Theory of Investment", American Economic Review, 50: 261-297.

Summers, L. (1981), "Taxation and Corporate Investment: A q-Theory Approach", Brookings Papers on Economic Activity, 1: 67-127.

Phelps, E. (1962), “The New View of Investment: A Neoclassical Analysis.", Quarterly Journal of Economics, 76: 548-567.

Tobin, J. (1969), “A General Equilibrium Approach to Monetary Theory”, Journal of Money, Credit and Banking, 1: 15-29.

Vergara, R. (2004), "Taxation and Private Investment: Evidence for Chile", mimeo, Pontificia Universidad Católica de Chile. 


\section{APENDICE}

Construcción del stock de capital

El stock de capital fue construido como sigue. Usamos el stock de capital inicial más la inversión en construcción, maquinarias y vehículos, excluyendo terrenos cuya información no está disponible para cada año de la muestra. Esta información nos permite escribir:

$$
K_{i t}^{S}=\left(K_{i(t+1)}^{S}-I_{i t}^{S}\right) /\left(1-\delta^{S}\right)
$$

donde

$S=$ obras de construcción (e), maquinaria $(m)$ o automóviles $(v)$

$K_{\text {it }}^{S}=$ stock de capital tipo s de la planta $\mathrm{i}$ en el año $\mathrm{t}$

$I_{i t}^{S}=$ inversión tipo s de la planta $\mathrm{i}$ en el año $\mathrm{t}$

$\delta^{S}=$ tasa de depreciación tipo $\mathrm{s}$

Asumimos las siguientes tasas de depreciación:

$\delta^{e}=$ construcción, ${ }^{6} 2 \%$

$\delta^{m}=$ maquinaria, ${ }^{6} 8 \%$

$\delta^{v}=$ vehículos, ${ }^{7} 15 \%$.

Para obtener el stock de capital por planta, sumamos todos los tipos de capital:

(A2) $\quad K_{i t}=K_{i t}^{e}+K_{i t}^{m}+K_{i t}^{v}$

Construcción del Producto Marginal del Capital

(A3) $P M K_{i t}=\left[S_{k}^{*}\left(V a_{i t} / K_{i t}\right)\right]-\delta_{i t}$

$S_{k}=$ participación del stock de capital, asumimos $40 \%$

$V a_{i t}=$ valor agregado en la planta ith en el año t

La tasa de depreciación es calculada como sigue:

\footnotetext{
${ }^{6}$ Ver Gutiérrez (1987).

${ }^{7}$ Ver Aguilar y Collinao (2001).
} 
(A4) $\delta_{i t}=\left[\left(E_{i t}^{*} \delta^{e}\right)+\left(M_{i t}^{*} \delta^{m}\right)+\left(V_{i t}^{*} \delta^{v}\right)\right] /\left(E_{i t}+M_{i t}+V_{i t}\right)$

Donde $E_{i t}, M_{i t}, V_{i t}$ es el stock de capital in construcción, maquinaria y vehículos de la planta $i^{\text {th }}$ en el año $t$, respectivamente. 
